\title{
Analysis on Mechanical Behavior of Acrylate Dielectric Elastomers
}

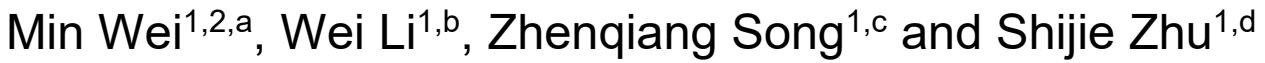

${ }^{1}$ Dept. of Intelligent Mechanical Engineering, Fukuoka Institute of Technology, Japan

${ }^{2}$ Dept. of Chemical Engineering, Nanjing University of Science and Technology, China

a648754735n@gmail.com, bluoyashang76@126.com, 'zqsong33@126.com, dzhu@fit.ac.jp

Keywords: Dielectric elastomer, Tensile behavior, Fatigue, Viscoelasticity.

\begin{abstract}
The dielectric elastomer (DE) has the advantages of large deformation ability, fast response speed, low price and high energy density. Therefore, the DE has great prospects as artificial muscle and flexible robot. The purpose of the research is to clarify the mechanical behavior for acrylate DE by tensile test, fatigue test and viscoelasticity measurement. The experimental results show that both the tensile strength and elongation increase with an increase in loading speed and the cyclic softening and energy loss occur during fatigue under both $0.5 \mathrm{~Hz}$ and $1 \mathrm{~Hz}$. The dependence of the $\tan \beta$ value on frequency was discussed.
\end{abstract}

\section{Introduction}

The DE as a kind of soft materials with flexible characteristics was widely applied to a variety of industries. Generally, characteristics like large strain, high stress, high energy density, great efficiency and fast response are required for the actuator applications. ${ }^{[1,2]}$ For example, under the control of mechanical force, ${ }^{[3]}$ it shows the action of great deformation and other features. For more than two decades, a variety of soft materials, including acrylate, silicone, chloroprene, urethane and thermoplastics have been used as DE materials for commercial applications and scientific research. The special requirements for soft materials depend on the actuator type and its foreseen application. ${ }^{[4]}$

The acrylate DEs from 3M company have been widely used for actuators, ${ }^{[5]}$ showing great performances with large actuator strain. However, their durability and viscoelasticity are not well understood for life prediction and mechanical design. This article aims at analyzing the relationship between stress and strain of acrylate DEs under tensile test, fatigue test and viscoelasticity test. The cyclic deformation and effects of frequency on viscoelasticity were studied for the materials screening for the actuator applications.

\section{Experiment Method}

\section{Materials}

The material used in this study was the acrylate DE (VBH Y-4905J, 3M Company). Its thickness is measured by the VHX-600 microscope. The thickness, optical features and molecular structure of material are shown in Table 1.

Table 1. Thickness, optical features and structure of materials

\begin{tabular}{cccc}
\hline Material & Thickness & Optical features & Structure \\
\hline Acrylate DE & $0.5 \mathrm{~mm}$ & Transparent & OH $_{\mathrm{OH}}$ \\
\hline
\end{tabular}

\section{Tensile test}

The shape and dimensions of the dumbbell shaped tensile specimen are shown in Fig. 1. The tensile test was conducted on the STA-1150 testing machine with the maximum stroke of $300 \mathrm{~mm}$. Under room temperature of about $26{ }^{\circ} \mathrm{C}$, specimens were stretched at the speeds of $100 \mathrm{~mm} / \mathrm{min}$, $300 \mathrm{~mm} / \mathrm{min}$ and $500 \mathrm{~mm} / \mathrm{min}$. 


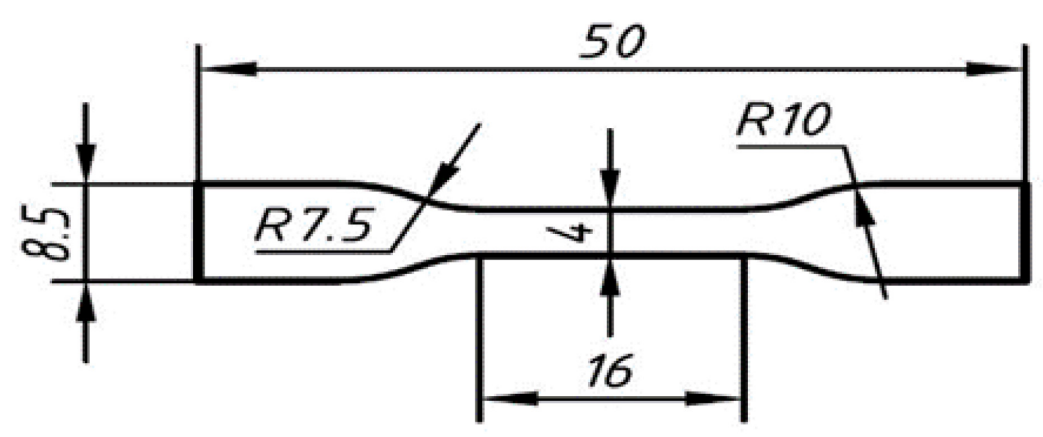

Fig. 1. Shape and dimensions of tensile specimens [mm]

\section{Fatigue test}

The shape and dimensions of the fatigue specimens are shown in Fig. 2, in which griping area was hatched. The Shimadzu MMT-101N fatigue testing machine was used for conducting fatigue tests under displacement control with a sine wave at room temperature $\left(26^{\circ} \mathrm{C}\right)$. The frequencies for fatigue tests were $0.5 \mathrm{~Hz}$ and $1 \mathrm{~Hz}$. The strain and stress were recorded up to 10,000 cycles at a strain range of $100 \%-200 \%$.

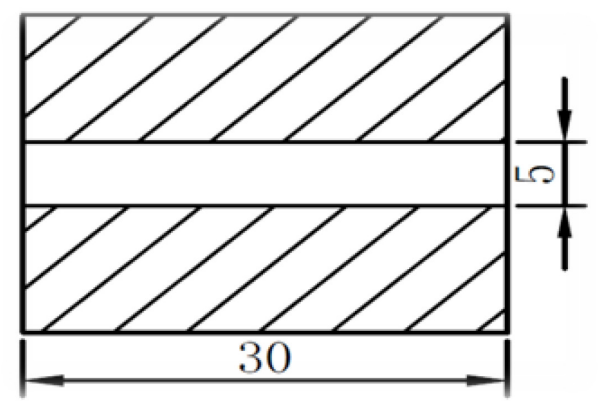

Fig. 2. Shape and dimensions of fatigue specimens [mm]

\section{Viscoelasticity test}

The specimens for viscoelasticity tests were disk shape with a diameter of $25 \mathrm{~mm}$. The viscoelasticity tests were carried out under shear loading with MCR302 rheometer (Anton Paar Company). The energy storage modulus and loss modulus were characterized as a function of frequency from 0.01 to $10 \mathrm{~Hz}$ under the rotation mode with $1 \%$ shear strain.

\section{Results and Analysis}

The stress versus strain curves of acrylic elastomer at the displacement rates of $100 \mathrm{~mm} / \mathrm{min}$, $300 \mathrm{~mm} / \mathrm{min}$ and $500 \mathrm{~mm} / \mathrm{min}$ are shown in Fig. 3. It can be seen that acrylic DE exhibits hyperplastic behavior. In particular, it is found that at the speed of $100 \mathrm{~mm} / \mathrm{min}$, the maximum stress of acrylate DEs is $8.97 \mathrm{MPa}$. In this case, within the strain range of 2 to 6 , the acrylate DE shows a plateau in the stress versus strain curves, which results in a very low secant modulus (Young's modulus). It is also found that under the same strain, the corresponding stress increases with the loading rate. Both the tensile strength (maximum stress) and elongation (maximum strain) increase with an increase in loading speed. 


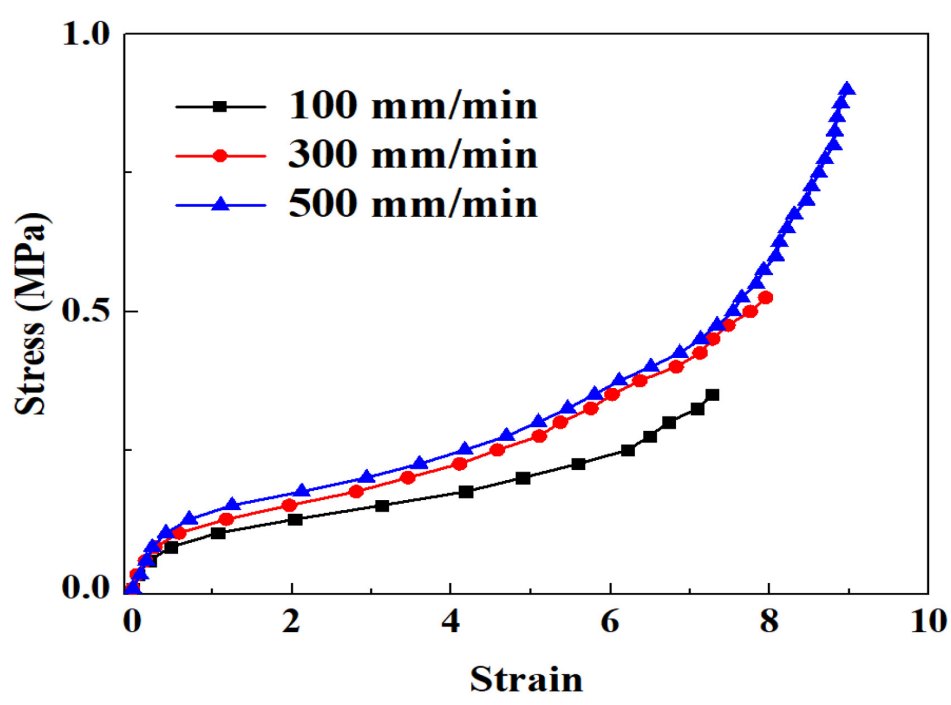

Fig. 3. Tensile stress versus strain curves of acrylate DE

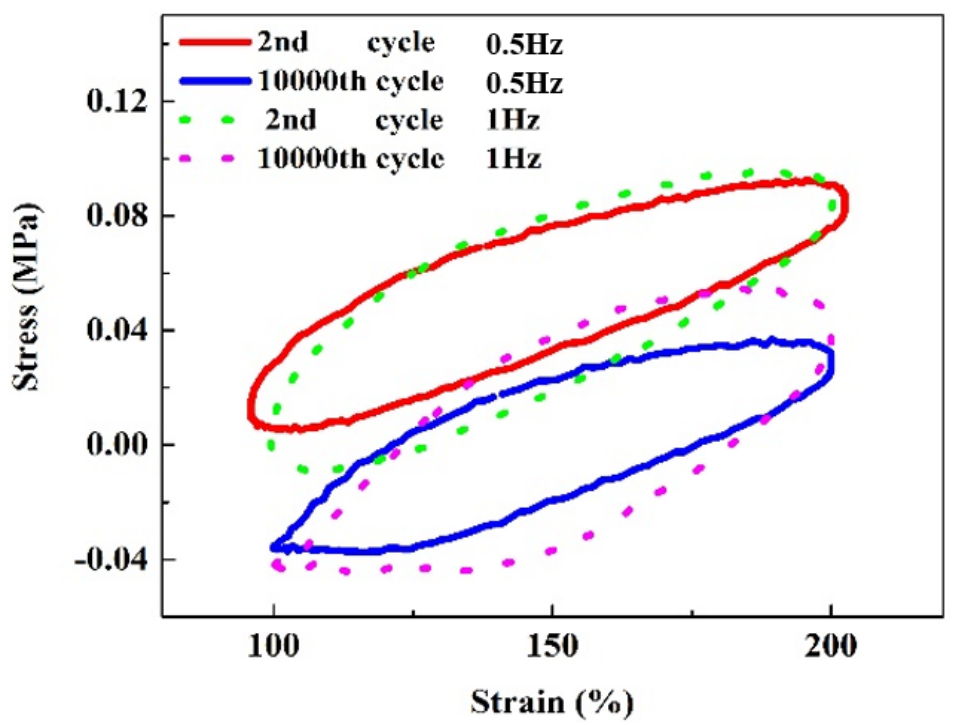

Fig. 4. Hysteresis loops of fatigue of Acrylate at $1 \mathrm{~Hz}$ and $0.5 \mathrm{~Hz}$

From Fig. 4, it is found that the hysteresis loop area of acrylate DE at $1 \mathrm{~Hz}$ is larger than $0.5 \mathrm{~Hz}$, which implies the higher energy loss for the former. After 10,000 cycles of loading, within the strain range of $100 \%-150 \%$, the stress range of $-0.04 \mathrm{MPa}$ to $-0.1 \mathrm{MPa}$ at $1 \mathrm{~Hz}$ does not exhibit significant changes, while from $0.5 \mathrm{~Hz}$ to $1 \mathrm{~Hz}$, the dissipated work of acrylate dielectric elastomer increases.

In Fig. 4, it also can be found that after 10,000 cycles of loading, the stress at the same strain is much lower than that of the 2nd cycle. This kind of softening phenomenon is much similar to the "Mullins effect", which was widely reported in the vulcanized rubber. ${ }^{[6]}$ In fact, the acrylic elastomer consists of a network of polymer chains, which are connected with multiple crosslinks. Polymer chains exhibit varying degree of flexibility, which are highly coiled at the unstretched state and extended along the applied stress direction before reaching fully stretched state. The crosslinks between the polymer chains exhibit different stretching limits, which might rupture at some weak points when the distance between neighboring chains exceed a certain value under the applied force. Subsequently, in the following loading process, the stress to sustain the same strain will be lower.

In the following equation, the $\tan \beta$ is calculated as the value of loss modulus $\left(G^{\prime \prime}\right)$ divided by storage modulus $\left(G^{\prime}\right)$, representing the relative value of energy loss. Materials with higher storage modulus show faster response to the stress, but the response time is also affected by the loss modulus.

$$
\tan \beta=G^{\prime \prime} / G^{\prime}
$$


From Fig. 5, both loss modulus $G^{\prime \prime}$ and storage modulus $G^{\prime}$ increase with the frequency, but the loss modulus exhibits a steeper trend than that of the storage modulus, which leads to the rise of $\tan \beta$. In essential, the $G^{\prime}$ is the ratio of the stress in phase with the stress to the strain, whereas $G^{\prime \prime}$ is the ratio of the stress $90^{\circ}$ out of the phase with the stress to the strain. Therefore, $G^{\prime}$ is directly proportional to the average energy storage in a cycle of deformation, while $G^{\prime \prime}$ is proportional to the average dissipation or loss of energy as heat in a cycle of deformation. Thus, the incremental value of $\tan \beta$ in Fig. 5 means that the energy dissipating dominates the material behavior as the frequency increase. This is in accordance with the fatigue testing results, as shown by the hysteresis loops in Fig. 4, where the area enclosed by the contour of stress-strain plot increases as the frequency increases from $0.5 \mathrm{~Hz}$ to $1 \mathrm{~Hz}$, especially after 10,000 cycles of loading. These results should provide significant clues for the design of $\mathrm{DE}$ actuators or generators with high energy conversion efficiency, which aims at reducing the mechanical work dissipation of the energy transducers.

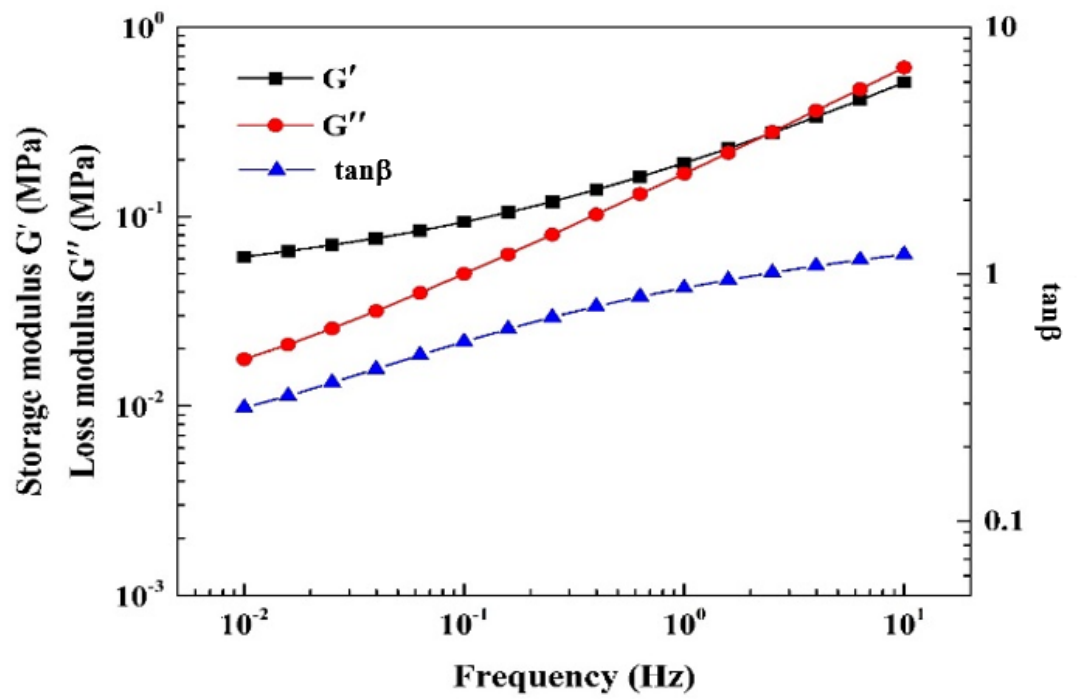

Fig. 5. The frequency dependence of $\tan \beta$ of acrylate dielectric elastomers

\section{Conclusion}

1) Both the tensile strength and elongation increase with an increase in loading speed.

2) Cyclic softening and energy loss were found during fatigue under both $0.5 \mathrm{~Hz}$ and $1 \mathrm{~Hz}$.

3) The $\tan \beta$ value increases with an increase in frequency.

\section{References}

[1] R. Pelrine, R. Kornbluh, G. Kofod, High-strain actuator materials based on dielectric elastomers. Adv. Mater., 12 (2000) 1223-1225.

[2] C. Cao, S. Burgess, A. Conn, T. Conn, Flapping at resonance: realization of an electroactive elastic thorax. In Proceedings of the IEEE/RAS RoboSoft 2018, Livorno, Italy, 24-28 April 2018.

[3] C. Laschi., M. Cianchetti, B. Mazzolai, L. Margheri, M. Follador, P. Dario, Soft robot arm inspired by the octopus. Adv. Robot., 7 (2012) 709-727.

[4] Q. Pei, M. Rosenthal, S. Stanford, H. Prahlad, R. Pelrine, Multiple-degrees-of-freedom electroelastomer roll actuators. Smart Mater. Struct., 5 (2004) 86.

[5] F. Ghazali, C. Mah, A. AbuZaiter, P. Chee, M. Ali, Soft dielectric elastomer actuator micropump. Sens. Actuators A Phys., 263 (2017) 276-284. 